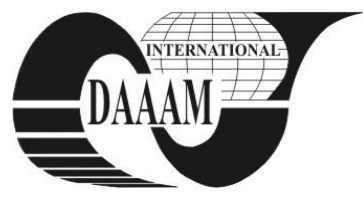

\title{
THE CONCENTRATION LEVEL OF TOURISTIC UNITS IN THE SUSTAINABLE DEVELOPMENT OF ROMANIAN TOURISM
}

\author{
ANDREI, D[aniela] R[uxandra]; GOGONEA, M[anuela] R[odica] \& STAN, E[lena] R[oxana]
}

\begin{abstract}
On international level in the development of tourism a tendency to reevaluate the environment in the conditions of sustainable and ecological development of touristic destinations, is noticed. In this context, the evolution of accommodation is analyzed by touristic destinations turning to medium and structural indicators and also degree of territorial concentration by applying territorial distribution coefficients. A correct evaluation of the number, of the touristic accommodation on the territory has a significant impact on establishing and the forecasting of the touristic request therefore on the results of the touristic activity, with deep implications on the sustainable development of tourism. The need to study the degree of territorial concentration of tourist facilities is a result of the impact of sustainable development of regional tourism on sustainable development in this field

Key words: accommodation, touristic destinations, degree of territorial concentration, sustainable development of tourism
\end{abstract}

\section{INTRODUCTION}

An essential component of the touristic offer is the establishment of touristic reception.

The oversized estimation process of the touristic potential of a touristic destination and the incorrect forecast regarding the touristic request can lead to the appearance of a excessive number of touristic establishments and so the creation of a unfavorable economic situation for those business owners, that will record an occupancy degree insufficient to ensure the nondeficiency of the business. On the other hand, there are oversaturated areas in which opening another accommodation unit would be, economically speaking, irrational and deficient.

The proposed analysis starts with an overall presentation of the evolution of accommodation made by touristic destinations, between the years of 1994 until 2010, strengthen by the results of the determined structural medium indicators (Gogonea, 2009). The calculated structural indicators (specific gravity: $g_{i}$ ) for the two years, 1994 and 2010, help in calculating the territorial concentration coefficients Gini and Struck (Badita, et al., 2002).

$$
\begin{gathered}
C_{G}=\sqrt{\sum_{i=1}^{n} g_{i}^{2}} \in\left[\sqrt{\frac{1}{n}} ; 1\right] \\
C_{S}=\sqrt{\frac{n \sum_{i=1}^{n} g_{i}^{2}}{n-1}} \in[0 ; 1]
\end{gathered}
$$

Determining the degree of variation of the distribution of territorial accommodation, in the presented context is essential for creating a suitable framework for territorial eco-touristic sustainable development, as a special component in this millennium.

\section{THE EVOLUTION OF ACCOMMODATION BY TOURISTIC DESTINATIONS}

The evolution tendency accommodation between 1994 and 2010 is synthetic presented through the six main touristic destinations (TD) of Romania (figure 1):

$>$ TD 1 - Seaside - In 1994 the Sun and the Black Sea hold an important place between the preferences of tourists; in this context continuing the construction process of the touristic units, the average established growth being of 19.06 units annually, representing an average increase of $2.26 \%$ with the hope that through a quality offer, oriented the "ecological" elements will satisfy at a high level the market's requests, the profit being high on local, regional, national, and international level.

$>$ TD 2 - Spas - they are the only destination the confronts an average decrease of 9.125 units, therefore $2 \%$, due to the pronounced lack of interest to evaluate this natural potential resource, that in other countries is very well exploited, especially that the existent offer involves the ecologic sustainable development;

$>$ TD 3 - Mountain - the attraction to the mountain air and heights, dressed as practice of tourism records a average growth of 26.875 units a year, which means that in relative value is represented by the $3.40 \%$, presented a satisfying evolution in the conditions of a sustainable development;

$>$ TD 4 - Danube Delta- the growth of the number of accommodation is moderately low in comparison with the other destinations, with only 4 units annually transposed relatively through an average increase of $4.48 \%$, which is pretty unsatisfying regarding the offer that could be significantly in the direction of ecologic in the context of sustainable development;

$>$ TD 5 - Residence towns- with a growing future, business tourism is the most to be exploited in this kind of destinations, fact seen in the average established growth of $7.52 \%$, which transposed in absolute value means an average increase of 45.625 units a year;

$>$ TD 6 - Other locations - developing and increasing the practice of cultural, religious and not lass business tourism, are transposed through the necessity of building new accommodation spaces; thus explaining the fact, that for this type of touristic destination records the highest average growth of 61.5 units annually, which means $6.53 \%$.

Overall, (fig. 1) the evolution of all the accommodation structures in Romania is confronted with a general growth tendency with a medium of $3.88 \%$, which means an average increase of 148.875 units a year.

The presented results describe the first level in the analysis of territorial repartition of touristic units. The next level, which will be dealt with next, will consist of a structural comparison of aforementioned units in the main touristic destinations. 


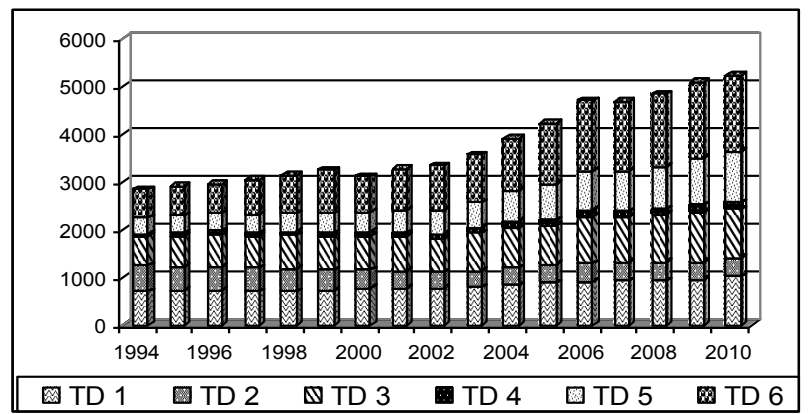

Fig. 1. Evolution of accommodation structures by destinations, between 1994 and 2010

The comparison of the accommodation structures numbers made by touristic destinations could also be highlighted structurally.

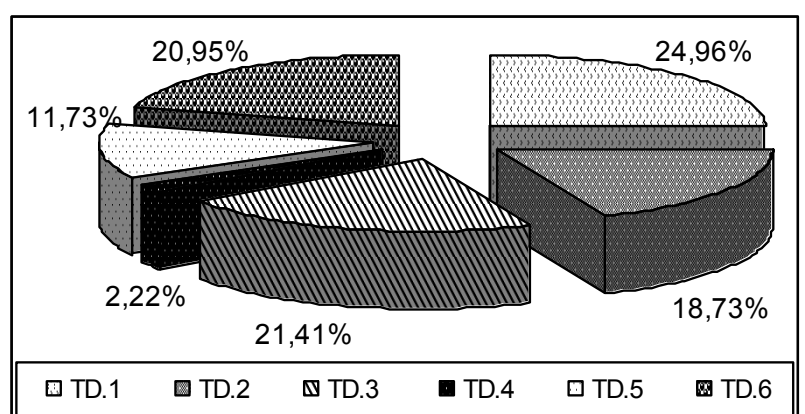

Fig. 2. The structure of accommodation structures made by destination in 1994

The presented conclusions regarding the evolution of accommodation structures are also highlighted through the accommodation structures in 2010 beside the ones in 1994.

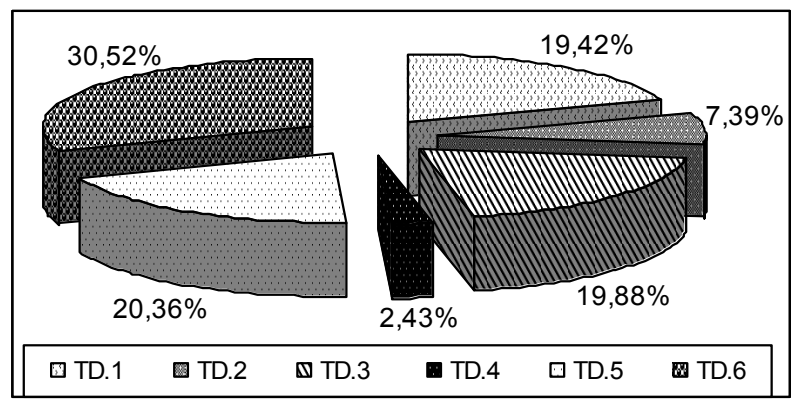

Fig. 3.The structure of accommodation structures made by destinations in 2010

Thereby the most significant increase is highlighted (graphics number 2 and 3) in other locations (TD 6), with a 9.574 percent difference, while the most obvious decrease is registered by spas (TD 2), with 11.34 percent.

\section{DETERMINING THE TERRITORIAL CONCENTRATION DEGREE OF ACCOMODATTION REGARDING TOURISTIC DESTINATIONS}

Given the fact that regional development of tourism has an important factor, the concentration degree accommodation units, we could say that it plays a decisive part in the analysis of the disparities between tourist destinations.

In this context the necessity of establishing the degree of territorial concentration by tourist destinations appears. This could be drawn starting from the structural coefficients (specific gravity), to get to apply the coefficients of Ginni $\left(\mathrm{C}_{\mathrm{G}}\right)$ and Struck $\left(\mathrm{C}_{\mathrm{S}}\right)$.
Because the results that are obtained through the method of Gini ( $\left.C_{G}=0,448\right)$ and Struck $\left(C_{S}=0,204\right)$, tend to the lower limits of the intervals $(0,408$, and 0$)$, reflect the tendency of uniformly distribute the accommodation structures by tourist destinations, in 1994.

The same tendency of uniformly distributed tourist structures is also established in 2010 for Romania. The coefficients values ( $C_{G}=0,466$ and $C_{S}=0,247$ ), being so close to the same lower limits of the intervals $(0,408$, and 0$)$ settle the fact mentioned for 2010.

\section{CONCLUSION}

Analysis of the territorial concentration of accommodation units by destinations began with the study of the evolution of accommodation in Romania, during 1994-2010. Thus it was established that the number of tourist facilities has increased in almost all tourist destinations, except the spa area which has experienced a significant decline. These trends of increase or decrease are also confirmed in structural terms by comparing the structures established in 2010 with the ones established in 1994.

Steady evolution is considered the first positive step in the analysis of territorial distribution of tourist facilities, which was completed by determining a relatively uniform distribution of them.

The low concentration degree, and the high uniformity represent a reflection of the regional development politics that was coordinated satisfying nationally through some wining project that were run between 1994 and 2010.

The differences, between regions, about the number of tourist structures are not highlighted fact that opens a satisfying perspective regarding the process of sustainable development of tourism.

The right evaluation, with a positive result, of the number of the distribution of tourist accommodation in the territory, has significant impact over establishing and forecasting the touristic request, therefore over the results of tourist activity, with practical and efficient implications over the sustainable development of tourism.

Establishing the degree of concentration for touristic units is a reference point for a future research of the uniform repartition impact of lodged tourists on the touristic activity from Romania.

\section{REFERENCES}

Gogonea, R.M. (2009). Statistics. Theoretical and practical basis for trade-services-tourism, Publisher Universitara, ISBN:978-973-749-588-4, Bucharest

Gogonea, R-M (2009). Trade activity-travel-services implemented in statistical applications, Publisher Universitara, ISBN:978-973-749-603-4, Bucharest

Badita, M.; Baron ,T. \& Cristache, S.E. (2002). Statistics for business and tourism trade (vol 1 \& 2), Publisher Luceafarul, ISBN:973-85399-749-5-1, Bucharest

Stan, E.R.; Gabroveanu, E.; Radneantu, N.; Andrei D.R. (2010). The influence of main quality factors on the evolution of the number of tourists - a study in a Romanian business hotel (volume 21), Published by DAAAM International Viena

Andrei, D.R.; Gogonea, R.M (2006), Measuring Tourism with the vision of sustainable development, Ed. Sedcom Libris, ISBN (10) 973-670-169-7, ISBN (13) 978-973-670-1696(CD), Iasi, and Tourism Magazine, nr.3/2007, cu ISSN:1844-2994, indexed in BDI Research Papers in Economics (SciSeach)

Gogonea, R.M; Andrei, D.R. (2006), The tourism`s implication in the process of economic growth, 3 rd International Scientific Conference ECO-TREND, Editura Universitaria Publishing House, Craiova 